\title{
Do Motion Controllers Make Action Video Games Less Sedentary? A Randomized Experiment
}

\author{
Elizabeth J. Lyons, ${ }^{1,2}$ Deborah F. Tate, ${ }^{1,2,3}$ Dianne S. Ward, ${ }^{1}$ Kurt M. Ribisl, ${ }^{3}$ \\ J. Michael Bowling, ${ }^{3}$ and Sriram Kalyanaraman ${ }^{4}$ \\ ${ }^{1}$ Department of Nutrition, The University of North Carolina at Chapel Hill, Chapel Hill, NC 27599-7461, USA \\ ${ }^{2}$ Lineberger Comprehensive Cancer Center, The University of North Carolina at Chapel Hill, Chapel Hill, NC 27599-7294, USA \\ ${ }^{3}$ Department of Health Behavior and Health Education, The University of North Carolina at Chapel Hill, Chapel Hill, \\ NC 27599-7440, USA \\ ${ }^{4}$ School of Journalism and Mass Communication, The University of North Carolina at Chapel Hill, Chapel Hill, \\ NC 27599-3365, USA
}

Correspondence should be addressed to Elizabeth J. Lyons, elyons@email.unc.edu

Received 5 May 2011; Revised 10 August 2011; Accepted 16 August 2011

Academic Editor: Hollie Raynor

Copyright ( $) 2012$ Elizabeth J. Lyons et al. This is an open access article distributed under the Creative Commons Attribution License, which permits unrestricted use, distribution, and reproduction in any medium, provided the original work is properly cited.

\begin{abstract}
Sports- and fitness-themed video games using motion controllers have been found to produce physical activity. It is possible that motion controllers may also enhance energy expenditure when applied to more sedentary games such as action games. Young adults $(N=100)$ were randomized to play three games using either motion-based or traditional controllers. No main effect was found for controller or game pair $(P>.12)$. An interaction was found such that in one pair, motion control (mean [SD] 0.96 $[0.20] \mathrm{kcal} \cdot \mathrm{kg}^{-1} \cdot \mathrm{hr}^{-1}$ ) produced $0.10 \mathrm{kcal} \cdot \mathrm{kg}^{-1} \cdot \mathrm{hr}^{-1}$ (95\% confidence interval 0.03 to 0.17 ) greater energy expenditure than traditional control $\left(0.86[0.17] \mathrm{kcal} \cdot \mathrm{kg}^{-1} \cdot \mathrm{hr}^{-1}, P=.048\right)$. All games were sedentary. As currently implemented, motion control is unlikely to produce moderate intensity physical activity in action games. However, some games produce small but significant increases in energy expenditure, which may benefit health by decreasing sedentary behavior.
\end{abstract}

\section{Introduction}

Sedentary screen time is a major public health problem. Sedentary behavior, particularly television (TV) watching, presents unique risks for obesity and related negative health outcomes in addition to risks from inactivity $[1,2]$; even individuals who are very active show increased metabolic risk with higher amounts of TV watching [3]. Sedentary video gaming has also been implicated as a predictor of obesity $[4,5]$. Video gaming using motion-based controllers has been proposed as a less sedentary alternative to TV watching or traditional video gaming [6], but these games vary widely in physical activity level produced during play. One factor that appears to have a large influence on energy expenditure is the type of controller used: for instance, dance and fitnessthemed games using dance mat and camera-based controllers consistently show higher levels of activity during play when compared to games that use more traditional gamepad controllers [6-11]. This difference is to be expected, as gamepads rely on small finger movements, whereas other control schemes engage large muscles in the arms and legs. Though dance and fitness-themed games are popular, action games remain the most widely played genre [12]. Action games typically emphasize skill, reflexes, and speed, and many of the most enduring video game franchises fall under this category; examples include games that require jumping across platforms (e.g., Super Mario Bros. and its many sequels) or precisely shooting or punching enemies (e.g., games in the Call of Duty or Street fighter series).

Integration of greater bodily motion into these traditional action video games may be a way to increase energy expended during play while retaining the features that make these games popular. Supplanting button presses for more realistic mimicking of, for example, punching or swordfighting would not necessarily change engaging aspects of these games (storyline, skill-testing gameplay, graphics, etc.). 
Motion control has even been hypothesized to potentially make games more engaging [13]. The Nintendo Wii console comes packaged with a Wiimote controller, which includes both buttons and motion-sensing functionality. Many action games can be played on the Wii console, but Wii versions of the games generally change the control scheme to include motion control for such actions as aiming a weapon or throwing grenades. It is as yet unclear whether these motions produce greater energy expenditure than typical button presses in traditional games. If motion controllers produce greater energy expenditure than traditional controllers, they could produce positive health impacts at both the individual and public health level.

The purpose of this study was to investigate the effects of motion-sensing controllers on energy expenditure during play of games from the action genre. Participants were randomized to play three games using either motion controllers or traditional controllers. The two games in each pair were different versions of the same game to minimize the presence of potential confounding variables. Enjoyment was also measured to investigate potential differences between controller groups. It was hypothesized that motion-controlled games would produce greater energy expenditure than the traditionally controlled games.

\section{Materials and Methods}

2.1. Study Participants and Protocol. Participants were 100 18-35-year olds, equal numbers of men and women, recruited primarily through a university online mailing list. The recruitment e-mail specifically solicited those interested in a video game research study. Participants were also recruited via a general weight-related study advertisement for 18-35-year olds shown on a local news network that directed interested viewers to a website with study descriptions and contact information. To be included, participants were required to weigh $<300$ pounds (necessary for the use of other game controllers in a larger study discussed elsewhere) [14], have played video games at least 3 times over the past year, be willing to fast 2.5 hours (necessary for indirect calorimetry), be videotaped during the study protocol, and have transportation to the study location. Of 757 individuals who requested information and eligibility criteria, 325 completed eligibility information; of those 325,169 potential participants were scheduled, and 100 completed the protocol. Eligible participants who did not attend their appointments $(N=49)$ were considered dropouts, 156 eligible participants were wait-listed, and 20 participated in a sub-study not reported here. All data were collected between April and August of 2009.

After providing informed consent, anthropometric measures (height, weight) were taken in light street clothes without shoes. Demographic variables were assessed via a preexperimental questionnaire. Participants were next led to a darkened video gaming laboratory and fitted with measurement equipment, then rested for 20 minutes. Controller type (traditional gamepad or motion Wiimote motion controller) was assigned randomly using a random number generator, and games were played in random order. Each game was played initially for a three-minute training period. All participants were provided with a visual aid to assist in learning controls, and study staff gave a brief introduction to the basic story and mechanics of the games. Once this period was complete, participants played for 10 minutes. Selfreport variables were measured immediately after play of each game by questionnaire. Additionally, participants were randomized to play several other types of games either before or after these games as part of a larger study [14]. Tests of the effects of game order and play of other games prior to this study on energy expenditure did not show significant effects (data not reported).

Games were played on a $58^{\prime \prime}$ high-definition television with optimized settings for each console. Participants sat in a gaming chair with speakers in the headrest that provided surround sound. The chair was placed approximately six feet from the television. Snack foods and drinks were available immediately after data collection concluded and water was available during rest periods. This protocol was approved by the University of North Carolina Public Health-Nursing Institutional Review Board.

2.2. Games. All participants played three games. Participants were randomized to play three games using either a buttonbased traditional gamepad controller or a motion-based Wiimote controller. All games were part of the broad genre of action games, rated $\mathrm{M}$ for Mature, and released between the years of 2002 and 2007.

Game pairs were chosen to represent different player perspectives, to investigate the effects of perspective and controller on psychological reactions not reported here. Figure 1 shows the study design. Medal of Honor: Heroes 2 and Medal of Honor: Airborne used a first-person perspective (i.e., players see through the eyes of their character), while Resident Evil 4: Wii Edition and Resident Evil 4 used an over-theshoulder perspective (players view the game environment from over the shoulder of their character). The final pair was chosen to vary both controller as well as perspective, and thus these two games were not as similar than the games in the other pairs. Resident Evil used a third-person perspective and a traditional controller, and Resident Evil: The Umbrella Chronicles used a first-person perspective and a motion controller.

The controllers used for the traditional play condition were the Playstation 3 (PS3) Dualshock controller and the Gamecube controller. Both controllers were of the traditional button-based gamepad type. The motion-sensing controllers used were the Wiimote and Nunchuk combination. The Wiimote, shaped like a remote control, was held in the right hand and used for both button and motion-based inputs. The Nunchuk was held in the left hand and used only for its button-based inputs.

2.3. Measures. Energy expenditure was measured via indirect calorimetry (Ultima CPX, MEDGRAPHICS, St. Paul, MN) using a neoprene mask and open Pneumotach. The indirect calorimeter was calibrated daily using a 3-liter syringe as well as prior to each test using certified gases. Oxygen consumption $\left(\mathrm{VO}_{2}\right)$ and carbon dioxide expiration $\left(\mathrm{VCO}_{2}\right)$ 


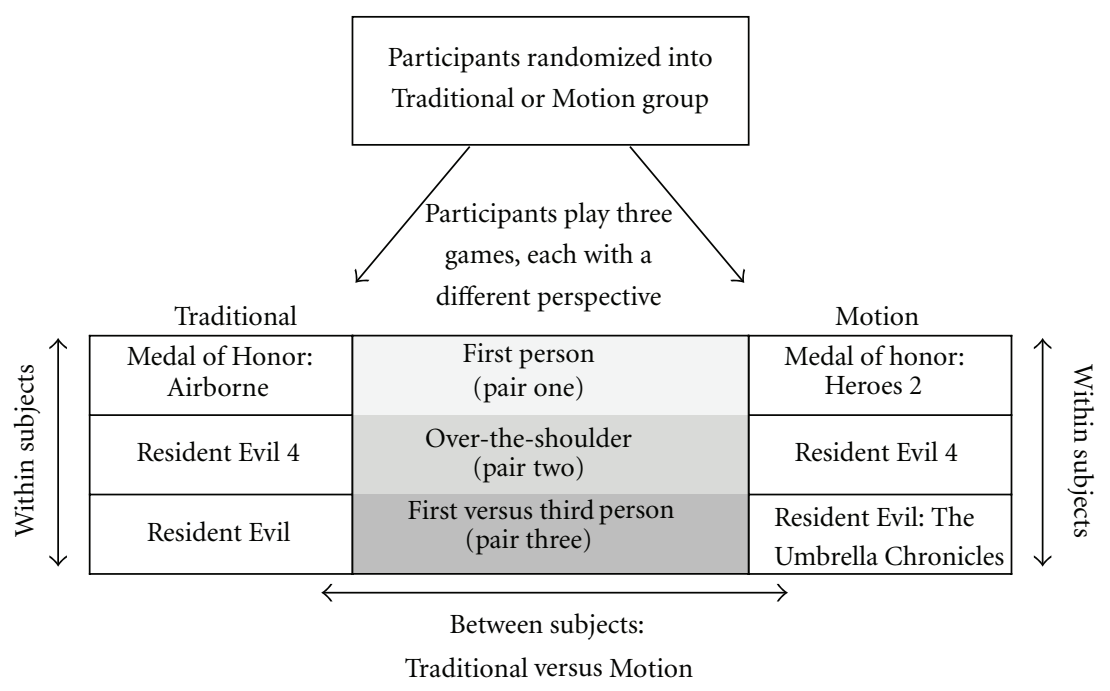

FIgure 1: Study design.

were measured on a breath-by-breath basis and converted to energy expenditure. Enjoyment was measured using the interest/enjoyment subscale of the Intrinsic Motivation Inventory, which is a well-validated measure that has been used in previous virtual reality physical activity studies $[15,16]$. This scale consisted of seven questions with responses on a Likert scale of 1-7. Participants were also asked about their general previous experience playing video games ("how much experience do you have playing video games?" with responses ranging from 1 , not a lot, to 7 , a lot) and whether they had played each of the games previously (dichotomous yes/no response). Weight and height were measured using a calibrated scale (Tanita, Arlington Heights, IL) and wall-mounted stadiometer (Perspective Enterprises, Inc., Kalamazoo, MI).

2.4. Data Preparation and Analysis. Energy expenditure data were averaged over the ten-minute play period and corrected for body mass ( $\mathrm{kcal} \cdot \mathrm{kg}^{-1} \cdot \mathrm{hr}^{-1}$, equal to metabolic equivalents or METs). Cut-points for sedentary behavior and light and moderate physical activity were taken from Pate et al. [17]. Energy expenditure <1.5 METs was considered sedentary. Two mixed models (dependent variables energy expenditure and enjoyment) were created, with game pair as a repeated measure and controller condition as an independent variable. Degrees of freedom were calculated using the Kenward-Roger method, and all tests of mean differences took into account row-wise degrees of freedom. Because several studies of energy expenditure during video game play have found effects of BMI and gender $[11,18]$, these variables were included in the model. The expenditure model was also run a second time with enjoyment as a covariate. Interaction terms for game pair $\times$ controller condition were also included. To investigate interactions, post hoc contrasts with Tukey-Kramer corrections were used. Games were contrasted within pairs and/or with the other games in their controller group (motion or traditional). Independent sample $t$-tests were also used for simple comparisons between groups, analyses of variance for tests of the impact of playing the game previously, and correlation analysis was used to investigate bivariate associations. The SAS software package (Cary, NC) version 9.2 was used for all analyses.

\section{Results}

Table 1 displays participant characteristics. The sample was 73\% White, 15\% Black, 8\% Asian, and 4\% other race. Six participants were of Hispanic ethnicity. Most participants were college graduates (49\%), followed by those with some college education (32\%). There were no significant differences between groups on any sociodemographic variables. Fifty-five percent of participants were overweight. Most participants had not played the games previously: 18 of the 100 had played Resident Evil, 25 had played Resident Evil 4, and 18 had played Medal of Honor. No differences in previous play were found by controller. No association was found between energy expenditure and video game playing experience or previous play of each game $(P>.05)$. Video game playing experience was positively associated with enjoyment (Resident Evil, $r=.255, P=.011$; Resident Evil 4, $r=.307, P=.002$; Medal of Honor, $r=.264, P=.008)$. Having ever played the game before predicted enjoyment in Resident Evil $(B=0.91, \mathrm{SE}=.63, P=.006)$ and Medal of Honor $(B=1.62, \mathrm{SE}=.63, P<.001)$ but not Resident Evil 4 $(B=0.54, \mathrm{SE}=.51, P=.101)$.

Mean energy expenditure corrected for body mass for each game is shown in Table 1. No main effect was found for controller $(P=.121)$ or game pair $(P=.510)$. However, an interaction between controller and game pair was found $(P=.004)$. The motion-controlled Resident Evil game (mean [SD] $0.96[0.20] \mathrm{kcal} \cdot \mathrm{kg}^{-1} \cdot \mathrm{hr}^{-1}$ ) produced 0.10 (95\% confidence interval [CI] $0.03-0.17, P=.048$ ) $\mathrm{kcal} \cdot \mathrm{kg}^{-1} \cdot \mathrm{hr}^{-1}$ greater energy expenditure than its comparison, the traditionally controlled Resident Evil game (0.86 [0.17] $\left.\mathrm{kcal} \cdot \mathrm{kg}^{-1} \cdot \mathrm{hr}^{-1}\right)$. Within the motion-controlled condition, the Resident Evil game produced 0.06 (95\% CI 0.02$0.11, P=.030) \mathrm{kcal} \cdot \mathrm{kg}^{-1} \cdot \mathrm{hr}^{-1}$ greater energy expenditure than Resident Evil $4\left(0.90[0.18] \mathrm{kcal} \cdot \mathrm{kg}^{-1} \cdot \mathrm{hr}^{-1}\right)$. No other 
TABle 1: Participant characteristics and energy expenditure and enjoyment outcomes by condition, mean (SD).

\begin{tabular}{|c|c|c|c|}
\hline Characteristic & Traditional $(N=50)$ & Motion $(N=50)$ & Total $(N=100)$ \\
\hline Age & $23.78(4.02)$ & $23.74(3.95)$ & $23.76(3.96)$ \\
\hline Height (cm) & $171.65(10.76)$ & $173.12(8.77)$ & $172.38(9.79)$ \\
\hline Weight (kg) & $79.44(20.65)$ & $81.37(20.74)$ & $80.40(20.62)$ \\
\hline $\operatorname{BMI}\left(\mathrm{kg} / \mathrm{m}^{2}\right)$ & $26.82(6.02)$ & $27.42(7.04)$ & $27.12(6.52)$ \\
\hline Experience & $5.80(1.36)$ & $5.90(1.21)$ & $5.85(1.28)$ \\
\hline \multicolumn{4}{|c|}{ Energy expenditure $\left(\mathrm{kcal} \cdot \mathrm{kg}^{-1} \cdot \mathrm{hr}^{-1}\right)$} \\
\hline Rest & $0.76(0.18)$ & $0.78(0.17)$ & $0.77(0.18)$ \\
\hline Resident Evil & $0.86(0.17)^{*}$ & $0.96(0.20)^{*}$ & $0.91(0.19)$ \\
\hline Resident Evil 4 & $0.90(0.17)$ & $0.90(0.18)$ & $0.90(0.18)$ \\
\hline Medal of Honor & $0.90(0.19)$ & $0.93(0.19)$ & $0.91(0.19)$ \\
\hline Overall (across game) & $0.89(0.16)$ & $0.93(0.17)$ & $0.91(0.16)$ \\
\hline \multicolumn{4}{|l|}{ Enjoyment } \\
\hline Resident Evil & $2.97(1.40)^{*}$ & $4.38(1.40)^{*}$ & $3.68(1.56)$ \\
\hline Resident Evil 4 & $4.29(1.38)^{* *}$ & $4.08(1.61)$ & $4.19(1.50)$ \\
\hline Medal of Honor & $4.48(1.74)^{* *}$ & $4.15(1.74)$ & $4.32(1.74)$ \\
\hline
\end{tabular}

cm: centimeter; kg: kilogram; m: meter; kcal: kilocalorie; hr: hour.

* Significant difference by controller, $P<.05$.

$* *$ Significantly different from traditionally controlled Resident Evil, $P<.001$

energy expenditure differences between games in a pair or within controller condition were found $(P>.30)$.

Participants with a higher BMI expended less energy during play than those with a lower BMI, B (SE) $=-0.01(0.00)$, $P<.001$. Energy expenditure did not differ by gender $(P=$ .645). All six games produced energy expenditure estimates below $1.5 \mathrm{kcal} \cdot \mathrm{kg}^{-1} \cdot \mathrm{hr}^{-1}$ (equal to METs), indicating that all were sedentary [17].

Analyses of enjoyment ratings found significant differences by game $(P<.001)$ as well as an interaction between game and console $(P<.001)$. The motion-controlled Resident Evil game produced a 1.39 (95\% CI 0.84-1.95) point higher enjoyment rating (on a scale of 1-7) than the traditionally controlled Resident Evil game. The other two traditionally controlled games were also rated as more enjoyable than the traditionally controlled Resident Evil game (Resident Evil 4, mean difference 1.32, 95\% CI 0.84-1.80; Medal of Honor, mean difference 1.51, 95\% CI 1.03-1.99). No other significant differences were found. Males $(B=1.19$, $\mathrm{SE}=0.20, P<.001)$ and those with higher BMI $(B=0.03$, $\mathrm{SE}=0.01, P=.028)$ rated the games as more enjoyable than females and those with lower BMI.

Inclusion of enjoyment as a covariate in the energy expenditure model slightly attenuated but did not alter the significance of the interaction between game pair and controller type $(P=.026)$. Enjoyment was not associated with energy expenditure in any of the game pairs $(P>.60)$, and this result did not differ by controller group.

\section{Discussion}

Contrary to our hypothesis, play of action games with motion-sensing controllers did not produce greater energy expenditure than play of similar games with traditional gamepad controllers. The traditional and motion-controlled games studied here produced average energy expenditures of 0.89 and $0.93 \mathrm{kcal} \cdot \mathrm{kg}^{-1} \cdot \mathrm{hr}^{-1}$, respectively, representing increases of 22 and 25 percent over rest measurements. In only one of three game pairs did the motion-controlled version produce significantly greater energy expenditure than a traditionally controlled version, a difference of $0.10 \mathrm{kcal} \cdot \mathrm{kg}^{-1} \cdot \mathrm{hr}^{-1}$. However, that game also produced greater energy expenditure than another of the motion-controlled games, suggesting that how motion controls are integrated into gameplay likely influences the intensity of motions used and thus energy expenditure.

Some motion-controlled games hold potential to significantly increase energy expenditure during screen time as compared to similar traditionally controlled games [19]. However, results of this study, showing only one of three games increasing expenditure, suggest that many action games likely do not. The results further suggest that the simple addition of motion control to traditional games, such as action games, is not sufficient to produce light or moderate intensity physical activity and will not consistently produce an improvement in activity intensity as compared to traditional controllers.

Replacement of traditional controllers with motion controllers during play of action games could positively affect health in several ways. Even very low intensity muscle contractions, such as those involved in standing, can prevent or negate metabolic changes that result from sedentary behavior $[20,21]$. Additionally, the muscle contractions necessary to play motion-controlled action games may interrupt longer stretches of sedentary gaming. Breaks in sedentary time, regardless of their length or intensity, are associated with lower waist circumference, BMI, triglycerides, and 2-hour plasma glucose independent of total sedentary time and moderate-vigorous activity [22]. In other words, even the smallest periods of very light intensity activity can be beneficial, particularly if they interrupt what would otherwise 
be a long session of sedentary behavior. Thus, the motions required for play of motion-based action video games, though not strenuous enough to produce light intensity activity, may be sufficient to reduce risk associated with sedentary screen time.

Previous studies have found that use of the Wiimote motion-sensing controller can produce light (1.5-3 METs) to moderate ( $>3 \mathrm{METs}$ ) intensity physical activity in several specific fitness- and sports-themed games (e.g., Wii Sports and Wii Fit), increasing energy expenditure over rest up to $328 \%[18,23]$. Though the Wii games studied here used the same controller as the games previously studied, due to our study design, they more closely resembled traditional sedentary games in both content and energy expenditure $[6,24]$. The motions encouraged by games like Wii Sports differ greatly from those encouraged by other Wii action games. Whereas Wii Sports encourages large, exaggerated movements such as swinging a tennis racket or punching an opponent, action games require greater precision of movement to ensure success. The game that showed the greatest energy expenditure in this study (Resident Evil: The Umbrella Chronicles) encouraged frequent shooting by offering unlimited ammunition for the default weapon. It may be that the abundance of ammunition (a rarity in action games, which often require management of scarce resources to increase suspense) allowed participants to shoot more often than they did in the other games and, thus, expend more energy. Though Resident Evil 4: Wii Edition, which produced significantly lower energy expenditure than The Umbrella Chronicles, also used motion controls, it required more precise shooting less often and placed a greater emphasis on exploration of the game environment, which did not require player motion. Even if motion controls are added to this genre of game, it may be that the characteristics of the genre itself generally discourage unnecessary movements.

The game that produced the lowest energy expenditure (traditionally controlled Resident Evil) was also rated less enjoyable than its comparison motion-controlled game as well as the other two traditionally controlled games. This finding was surprising, as Resident Evil is considered a classic game, and the version used (the 2002 Gamecube remake) received extremely high review scores. Enjoyment was also predicted by overall gaming experience and having played the game before in Resident Evil and Medal of Honor games. It is unclear whether previous experience leads to increased enjoyment due to familiarity or if previous play and enjoyment both reflect an underlying variable such as a preference for specific types or series of games.

A strength of this study was the experimental study design and inclusion of three different pairs of games. To our knowledge, this study was the largest one of motion-controlled gaming yet conducted, with a much larger proportion of female participants (50\%) than in past studies. As women represent approximately $40 \%$ of video game players [12], their inclusion in active gaming studies is important. Participants were randomized to play iterations of the same game using different controllers, eliminating many potential confounding variables related to game content that may have biased previous studies.
A limitation of the study was that only one of the three pairs included identical games. The other two pairs included games that were chosen to minimize potential confounding variables; however, it was impossible to eliminate them. The pair that produced significant differences in energy expenditure, Resident Evil and Resident Evil: The Umbrella Chronicles, was the least similar of the three pairs. The games shared characters, storyline, and setting, but differences in implementation of control scheme may have led to differences in gameplay. Differences in this pair (e.g., more frequent shooting, greater enjoyment), offer insight into possible variables to manipulate in future studies that may produce increases in energy expenditure.

Other limitations include the cross-sectional nature of the data and measurement of only one side of energy balance. It is impossible based on this study design to determine potential effects of game play on subsequent energy intake and expenditure. Play of video games with a traditional controller has been found to increase food consumption after a play session [25]; thus, it is possible that later increases in energy intake could erase the expenditure benefits of motion-controlled action gaming and result in overall positive energy balance. Results should also be generalized with caution due to possible differences between eligible participants who completed the study and those who did not attend their appointment or who were eligible but not asked to participate because recruitment goals (i.e., 100 participants, 50 per gender) were reached.

\section{Conclusions}

Action video games are extremely popular and are the most widely played genre of video game. Addition of motion controls to this genre of game can produce small increases in energy expended during play. However, only some motioncontrolled action games produce greater expenditure than equivalent traditionally controlled games; these games also produce greater energy expenditure than some other motion-controlled games. The manner in which motions are integrated into gameplay likely has a large effect on the amount of energy expenditure than can be expected during play. Though motion-controlled action games may not lead to changes in the intensity of physical activity, their potential for reducing risk associated with sedentary behavior should not be overlooked.

Little is currently known about the impact of motioncontrolled games on sedentary behavior and physical activity over time. Even less is known about the impact of these games on other behaviors that may be associated with obesity and other negative health outcomes, such as snacking during screen time. There is a particular need for investigation of game characteristics that encourage or discourage movement even in highly similar games, such as the two motion-controlled Resident Evil games played in this study that produced significantly different energy expenditure levels. Randomized controlled trials are necessary to investigate the potential of games to contribute to obesity prevention and treatment; even games that do not increase moderate-vigorous intensity 
physical activity may improve health by decreasing prolonged bouts of sedentary behavior.

\section{Conflict of Interests}

The authors declare that they have no competing interests.

\section{Authors' Contributions}

E. J. Lyons and D. F. Tate designed the study and obtained funding. E. J. Lyons, D. F. Tate, and J. M. Bowling planned and performed statistical analyses, and all authors participated in interpretation of analyses. E. J. Lyons drafted the paper. D. F. Tate, D. S. Ward, K. M. Ribisl, J. M. Bowling, and S. Kalyanaraman critically revised the paper. All authors read and approved the final paper.

\section{Acknowledgments}

The authors thank Phillip Carr and Stephanie Komoski for their assistance in data acquisition and cleaning; Kristen Polzien, Ph.D. for her assistance with energy expenditure measurement and analysis; and Karen Erickson, M.P.H., R.D. for her assistance with study administration. This study was funded by the Robert Wood Johnson Foundation's Health Games Research Initiative grant number 64438 and by Lineberger Comprehensive Cancer Center's Cancer Control Education Program, which is funded by National Cancer Institute grant number CA57726.

\section{References}

[1] D. W. Dunstan, J. Salmon, G. N. Healy et al., "Association of television viewing with fasting and 2 -h postchallenge plasma glucose levels in adults without diagnosed diabetes," Diabetes Care, vol. 30, no. 3, pp. 516-522, 2007.

[2] U. Ekelund, S. Brage, K. Froberg et al., "TV viewing and physical activity are independently associated with metabolic risk in children: the European youth heart study," PLoS Medicine, vol. 3, no. 12, Article ID e488, pp. 2449-2457, 2006.

[3] G. N. Healy, D. W. Dunstan, J. Salmon, J. E. Shaw, P. Z. Zimmet, and N. Owen, "Television time and continuous metabolic risk in physically active adults," Medicine and Science in Sports and Exercise, vol. 40, no. 4, pp. 639-645, 2008.

[4] M. M. Carvalhal, M. C. Padez, P. A. Moreira, and V. M. Rosado, "Overweight and obesity related to activities in Portuguese children, 7-9 years," European Journal of Public Health, vol. 17, no. 1, pp. 42-46, 2007.

[5] M. Schneider, G. F. Dunton, and D. M. Cooper, "Media use and obesity in adolescent females," Obesity, vol. 15, no. 9, pp. 2328-2335, 2007.

[6] L. Lanningham-Foster, T. B. Jensen, R. C. Foster et al., "Energy expenditure of sedentary screen time compared with active screen time for children," Pediatrics, vol. 118, no. 6, pp. e1831e1835, 2006.

[7] R. Maddison, C. Ni Mhurchu, A. Jull, Y. Jiang, H. Prapavessis, and A. Rodgers, "Energy expended playing video console games: an opportunity to increase children's physical activity?" Pediatric Exercise Science, vol. 19, no. 3, pp. 334-343, 2007.
[8] L. Straker and R. Abbott, "Effect of screen-based media on energy expenditure and heart rate in 9- to 12-year-old children," Pediatric Exercise Science, vol. 19, no. 4, pp. 459-471, 2007.

[9] B. Tan, A. R. Aziz, K. Chua, and K. C. Teh, "Aerobic demands of the dance simulation game," International Journal of Sports Medicine, vol. 23, no. 2, pp. 125-129, 2002.

[10] K. Sell, T. Lillie, and J. Taylor, "Energy expenditure during physically interactive video game playing in male college students with different playing experience," Journal of American College Health, vol. 56, no. 5, pp. 505-511, 2008.

[11] V. B. Unnithan, W. Houser, and B. Fernhall, "Evaluation of the energy cost of playing a dance simulation video game in overweight and non-overweight children and adolescents," International Journal of Sports Medicine, vol. 27, no. 10, pp. 804-809, 2006.

[12] Entertainment Software Association, "Essential facts about the computer and video game industry," 2010, http://www.theesa .com/facts/pdfs/ESA_Essential_Facts_2010.PDF.

[13] M. Slater, A. Steed, J. McCarthy, and F. Maringelli, "The influence of body movement on subjective presence in virtual environments," Human Factors, vol. 40, no. 3, pp. 469-477, 1998.

[14] E. J. Lyons, D. F. Tate, D. S. Ward, J. M. Bowling, K. M. Ribisl, and S. Kalyararaman, "Energy expenditure and enjoyment during video game play: differences by game type," Medicine and Science in Sports and Exercise, vol. 43, no. 10, pp. 19871993, 2011.

[15] W. A. IJsselsteijn, Y. A. W. de Kort, J. Westerink, M. De Jager, and R. Bonants, "Virtual fitness: stimulating exercise behavior through media technology," Presence-Teleoperators and Virtual Environments, vol. 15, no. 6, pp. 688-698, 2006.

[16] E. McAuley, T. Duncan, and V. V. Tammen, "Psychometric properties of the intrinsic motivation inventory in a competitive sport setting: a confirmatory factor analysis," Research Quarterly for Exercise and Sport, vol. 60, no. 1, pp. 48-58, 1989.

[17] R. R. Pate, J. R. O’Neill, and F. Lobelo, “The evolving definition of "sedentary"', Exercise and Sport Sciences Reviews, vol. 36, no. 4, pp. 173-178, 2008.

[18] L. Graves, G. Stratton, N. D. Ridgers, and N. T. Cable, "Energy expenditure in adolescents playing new generation computer games: cross sectional study," British Medical Journal, vol. 335, no. 7633, pp. 1282-1284, 2007.

[19] A. L. Penko and J. E. Barkley, "Motivation and physiologic responses of playing a physically interactive video game relative to a sedentary alternative in children," Annals of Behavioral Medicine, vol. 39, no. 2, pp. 162-169, 2010.

[20] T. W. Zderic and M. T. Hamilton, "Physical inactivity amplifies the sensitivity of skeletal muscle to the lipid-induced downregulation of lipoprotein lipase activity," Journal of Applied Physiology, vol. 100, no. 1, pp. 249-257, 2006.

[21] M. S. Tremblay, R. C. Colley, T. J. Saunders, G. N. Healy, and N. Owen, "Physiological and health implications of a sedentary lifestyle," Applied Physiology, Nutrition and Metabolism, vol. 35, no. 6, pp. 725-740, 2010.

[22] G. N. Healy, D. W. Dunstan, J. Salmon et al., "Breaks in sedentary time: beneficial associations with metabolic risk," Diabetes Care, vol. 31, no. 4, pp. 661-666, 2008.

[23] D. L. Graf, L. V. Pratt, C. N. Hester, and K. R. Short, "Playing active video games increases energy expenditure in children," Pediatrics, vol. 124, no. 2, pp. 534-540, 2009. 
[24] P. Borusiak, A. Bouikidis, R. Liersch, and J. B. Russell, "Cardiovascular effects in adolescents while they are playing video games: a potential health risk factor?" Psychophysiology, vol. 45, no. 2, pp. 327-332, 2008.

[25] J. P. Chaput, T. Visby, S. Nyby et al., "Video game playing increases food intake in adolescents: a randomized crossover study," American Journal of Clinical Nutrition, vol. 93, no. 6, pp. 1196-1203, 2011. 


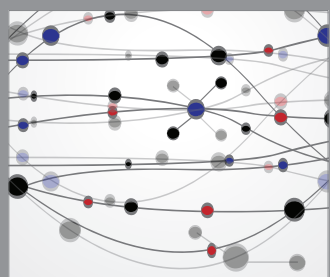

The Scientific World Journal
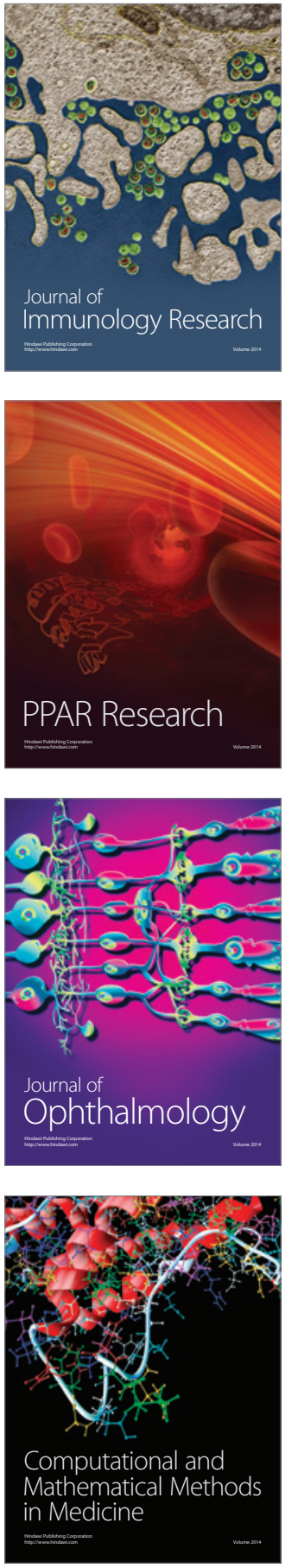

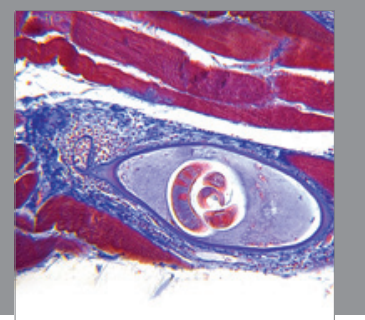

Gastroenterology

Research and Practice
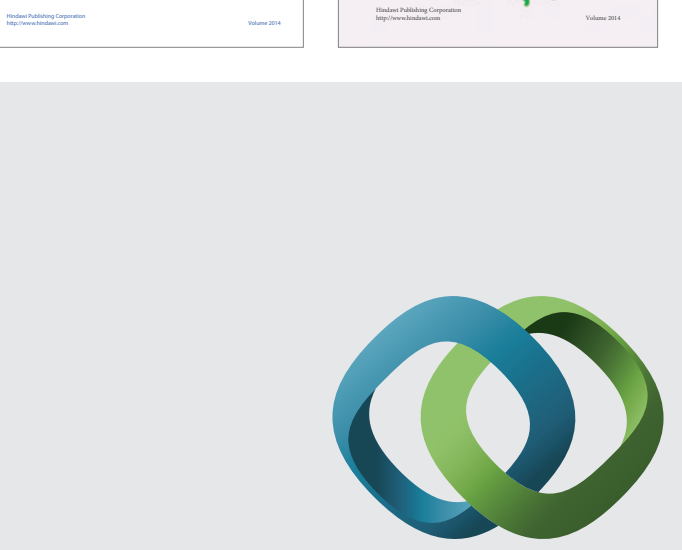

\section{Hindawi}

Submit your manuscripts at

http://www.hindawi.com
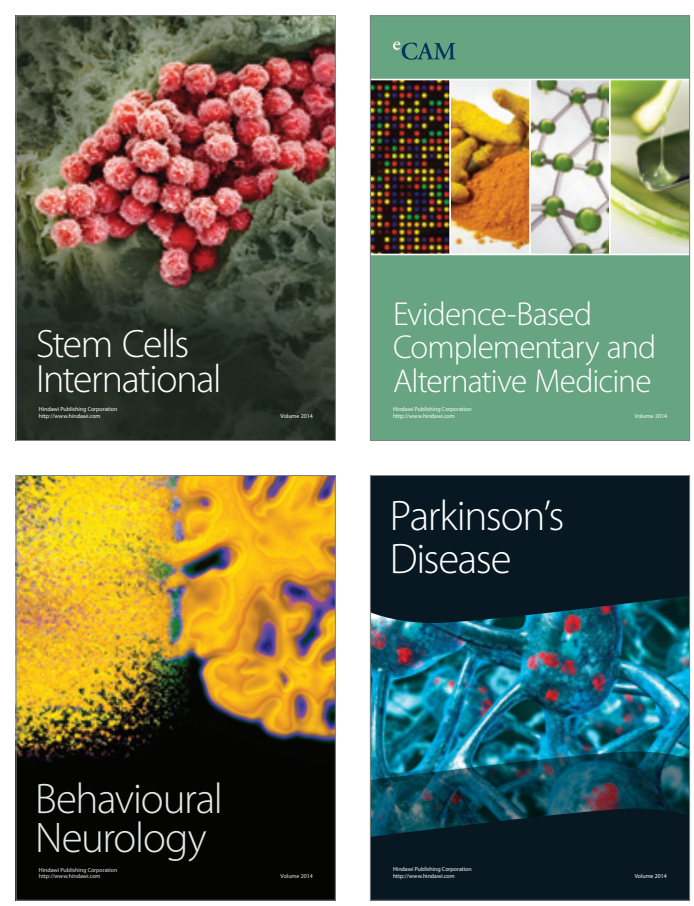

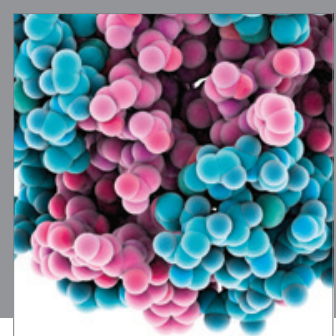

Journal of
Diabetes Research

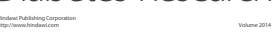

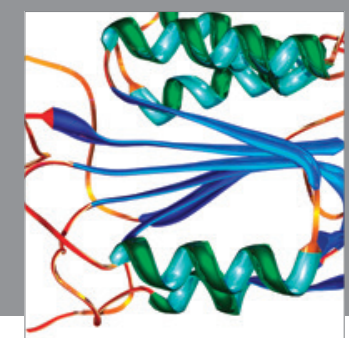

Disease Markers
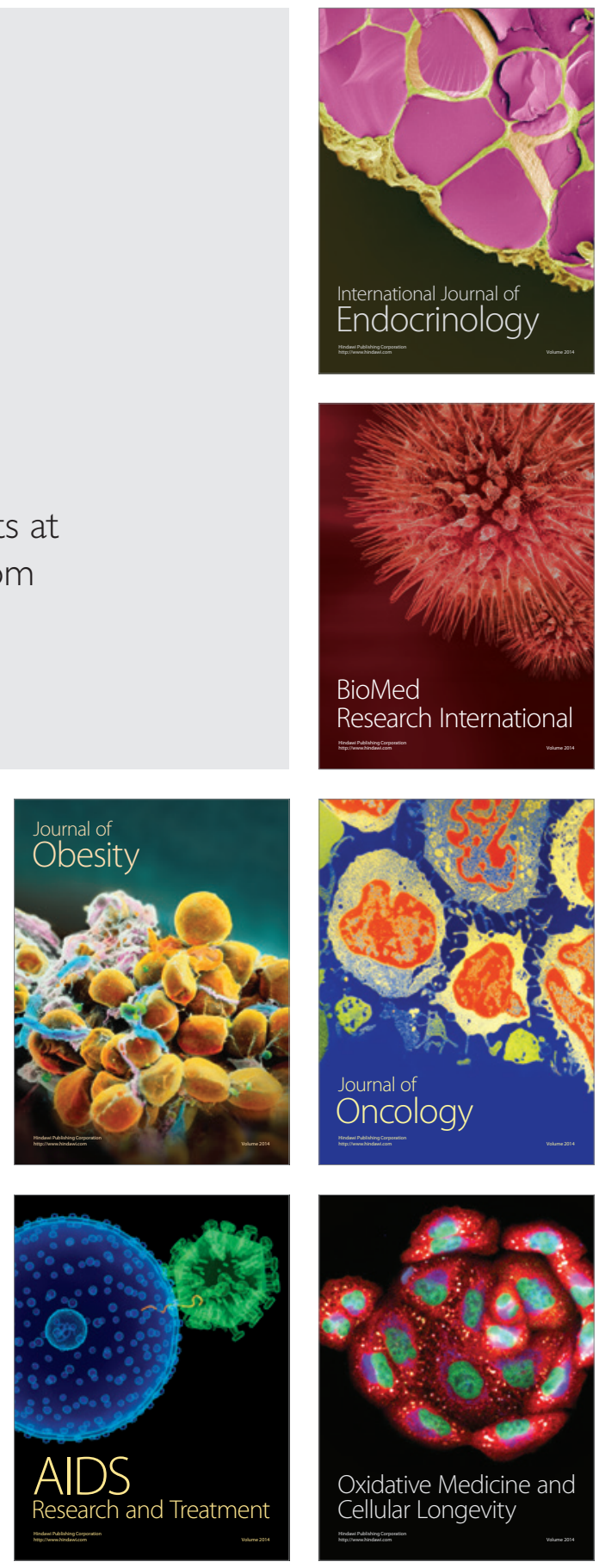